\title{
Efecto del contenido de titanio en la porosidad y dureza de un compuesto Cu-Ti-TiC sinterizado por descarga luminiscente anormal
}

\section{Effect of titanium content on the porosity and hardness of $\mathrm{Cu}$-Ti-TiC composite sintered by abnormal glow discharge}

Cristian Daniel Bohorquez Moreno ${ }^{1}$, Sandra Patricia Pérez Velásquez ${ }^{1}$, Armando Sarmiento Santos ${ }^{2}$, Martin Emilio Mendoza Oliveros ${ }^{1}$

\footnotetext{
${ }^{1}$ Grupo de Investigación en Materiales Siderúrgicos, Universidad Pedagógica y Tecnológica de Colombia, Tunja, Boyacá, Colombia.

${ }^{2}$ Grupo de Superficies, Electroquímica y Corrosión, Universidad Pedagógica y Tecnológica de Colombia, Tunja, Boyacá, Colombia.

e-mail: cristiandaniel.bohorquez@uptc.edu.co,patricia.perez@uptc.edu.co, asarmiento.santos@uptc.edu.co, martin.mendoza@uptc.edu.co
}

\section{RESUMEN}

Se ha sintetizado y caracterizado un material compuesto de matriz metálica (MMC) base cobre, reforzado con carburo de titanio al $1 \%(\% \mathrm{w} / \mathrm{w})$ y titanio en fracciones de masa del $10 \%, 15 \%$ y $20 \%$, con el fin de evaluar la porosidad y dureza en el material generada por la presencia del titanio en la matriz metálica. El MMC fue manufacturado por medio del proceso pulvimetalúrgico que comprendió: mezclado mecánico y ultrasónico de polvos de $\mathrm{Cu}$-Ti-TiC en suspensión liquida de 2-propanol, posterior secado y compactación uniaxial en frío a $400 \mathrm{MPa}$, sometiéndolo a sinterización por descarga luminiscente anormal a $850{ }^{\circ} \mathrm{C}$, empleando una atmósfera de $10 \%$ de nitrógeno y $90 \%$ de argón con un tiempo de sinterizado de 30 minutos. Los MMC fueron caracterizados estructuralmente por difracción de rayos $\mathrm{X}$ con el fin de determinar las fases presentes. La caracterización morfológica y composicional fue realizada por microscopia electrónica de barrido (SEM) y espectroscopía de energías dispersas de rayos (EDS). La porosidad en verde y de los sinterizados fue estimada por medio de SEM, seguida de análisis digital de imágenes. Como resultado se evidencia que los MMC en verde exhibieron una porosidad en el rango de 5,20\% y 7,21\% en las tres fracciones de Ti y en los sinterizados se obtuvieron valores cercanos al 13,6 \% con concentraciones del $20 \%$ de titanio. El análisis de difracción de rayos X, evidenció la presencia mayoritaria de las fases $\mathrm{Cu}_{3} \mathrm{Ti}_{\text {y }} \mathrm{CuTi}_{2}$ a $850{ }^{\circ} \mathrm{C}$. Las imágenes por SEM y EDS mostraron la distribución de titanio asociada a fenómenos difusivos durante la sinterización. La dureza fue determinada con el empleo de un microdurómetro con una carga de 100 gf, mostrando que el incremento de los contenidos de titanio, ha influenciado en la dureza debido a la presencia de fases intermetálicas, obteniéndose valores de hasta $454 \mathrm{HV}$.

Palabras clave: compuestos, intermetálicos, descarga luminiscente anormal, porosidad, Cu-Ti-TiC.

\section{ABSTRACT}

Copper metal matrix composites reinforced with $\mathrm{TiC} 1 \%(\% \mathrm{w} / \mathrm{w})$ and titanium mass with fractions of $10 \%$, $15 \%$ and $20 \%$ were synthesized and characterized in order to evaluate the porosity in the material generated by the presence of titanium and material hardness. The MMC's were manufactured by powder metallurgical route that included: mechanical and ultrasonic mixing of $\mathrm{Cu}$-Ti-TiC in liquid suspension of 2-propanol, following by drying with subsequent uniaxial cold compaction at $400 \mathrm{MPa}$. Sintering by abnormal glow discharge at $850{ }^{\circ} \mathrm{C}$ in $10 \%$ nitrogen and $90 \%$ argon atmosphere; sintering time of 30 minutes and later cooling. Sintered samples were characterized by X-ray diffraction to determine the material phases. The morphological and compositional characterization were performed by scanning electron microscopy (SEM) equipped with energy dispersive spectrometer (EDS). Porosity in green and sintered conditions were 
estimated by digital image analysis. As a result, in the green samples were evidenced a porosity in the range between $5,2 \%$ and $7,21 \%$ with the three fractions of $\mathrm{Ti}$. The sintered samples value close to $13,6 \%$ were obtained with concentrations of $20 \%$ of Ti. The X-ray diffraction analysis showed the majority presence of the $\mathrm{Cu}_{3} \mathrm{Ti}$ and $\mathrm{CuTi}_{2}$ phases at $850{ }^{\circ} \mathrm{C}$. The results by SEM and EDS exhibited the distribution of titanium associated with diffusive phenomena during sintering process. The hardness data were determined with the use of Vickers microdurometer with a load of $100 \mathrm{gf}$, showing that the presence of higher titanium content had influenced the hardness due to the formation of intermetallic phases, increasing its values up to $454 \mathrm{HV}$.

Keywords: composites, intermetallics, abnormal glow discharge, porosity, $\mathrm{Cu}-\mathrm{Ti}-\mathrm{TiC}$.

\section{INTRODUCCIÓN}

El cobre es considerado como uno de los materiales con mayor impacto en el mundo, ha acompañado el paso tecnológico del hombre desde hace siglos y se proyecta en los próximos años como un metal estratégico. Se estima que para el 2020 la demanda de cobre estará por encima de los 20 millones de toneladas [1], convirtiéndolo en un metal valorado, relativamente económico en su producción, así como, su fácil reciclaje, posibilitándolo como un material sustentable de consumo. Sus atractivas propiedades como: alta conductividad eléctrica, térmica, alta resistencia a la corrosión, lo hacen atractivo para una variedad de aplicaciones, entre ellas: la elaboración de cables, contactos eléctricos, intercambiadores de calor e inclusive objetos decorativos, debido a su agradable apariencia. No obstante, su uso como elemento estructural está restringido en razón a sus bajas propiedades mecánicas.

La síntesis de materiales compuestos de matriz metálica (MMC) busca dar una solución a las bajas características mecánicas del cobre, por medio de la incorporación de agregados (una o más fases) de distintos tipos de materiales como: metales (hierro y acero inoxidable) [2], [3], cerámicos del tipo $\mathrm{Al}_{2} \mathrm{O}_{3}$ [4], $\mathrm{AlN}$ [5], $\mathrm{TiB}_{2}, \mathrm{WC}, \mathrm{SiO}_{2}$, grafito [6] y TiC [7], [8], [9], [10], [11]. Este último ha despertado interés en la comunidad científica debido a las propiedades resultantes en combinación con materiales de matriz de cobre, su uso en fracciones másicas de $1 \%$ ha resultado en una mejora en la dureza, y por consiguiente en las propiedades mecánicas finales que exhibe el MMC. Sin embargo, la poca mojabilidad con la matriz expone resultados poco satisfactorios frente a la resistencia mecánica, debida a zonas frágiles dentro del sistema matriz-cerámica.

Se espera que con la incorporación de una segunda fase metálica dentro de la matriz mejoren propiedades como la dureza y resistencia a la tracción en especial si la fase cerámica pertenece al mismo grupo que la especie metálica. En este sentido, para compuestos reforzados con TiC, el uso de titanio se presenta como un buen candidato a ser incorporado en un MMC. El titanio es el séptimo elemento más abundante en la naturaleza, cuyas aplicaciones se están ampliando en diversas áreas de la industria. Su combinación con una matriz metálica como el cobre; ha sido estudiada por exhibir atrayentes propiedades que ofrecen las fases intermetálicas que se forman entre estos elementos [12].

Bajo condiciones de síntesis convencional, la aparición de fases intermetálicas está llamando cada vez más la atención debido a que por sus propiedades pueden ser aplicadas para resolver problemas de tipo mecánico, en particular en el área biomédica en donde influye el factor de producción, ya que piezas hechas de titanio puro representan un alto costo. Por otro lado, el cobre permite obtener partes a un menor valor con propiedades equivalentes, además, en otras áreas donde se prevé una aplicación es en el remplazo paulatino de las aleaciones $\mathrm{Cu}$-Be a causa de su alta toxicidad [13]. Esto hace necesario una transición a aleaciones más seguras que presenten una adecuada respuesta ingenieril [14] y condiciones de síntesis más adecuadas como las previstas en el sistema $\mathrm{Cu}-\mathrm{Ti}$.

Se hace necesario estudiar diferentes adiciones de Ti en materiales pulvimetalúrgicos dentro de la matriz de cobre en virtud de la emergente necesidad de desarrollar productos económicos empleando materiales ya conocidos en las industrias. Se ha determinado que la presencia de titanio en la matriz de cobre exhibe una modificación en la conductividad térmica de hasta $752 \mathrm{~W} . \mathrm{m}^{-1} \cdot \mathrm{K}^{-1}$ en comparación al cobre puro [15]. Estos resultados sugieren que la influencia de las adiciones de titanio debe seguir estudiándose con el fin de mantener una alta conductividad térmica y eléctrica en la matriz de cobre, así como mejorar su respuesta mecánica.

La pulvimetalúrgia ha brindado soluciones para el desarrollo de nuevos materiales, los cuales no podrían obtenerse por métodos tradicionales como la fusión y colado, que en particular para las aleaciones cobre-titanio son de alto costo. Esto abre un abanico de posibilidades de las propiedades que se logran obtener ya que dependiendo del método de sinterizado se afectan las características finales de los compuestos pulvimetalúrgicos como el tamaño de grano, dureza, porosidad y densidad. El uso de la sinterización por 
medio de técnicas de flash sintered frente a la metodología tradicional, es de gran interés en la industria moderna, es por ello que técnicas como el Spark Plasma Sintering ha permitido obtener resultados mecánicos interesantes en los sinterizados, como lo muestran los reportes de Wang et al [16], [17], Moraru et al [18], Samal et al [19].

Sin embargo, los altos costos del equipo imposibilitan el uso extensivo de este tipo de técnicas, a causa de esto, la descarga luminiscente anormal (DLA) surge como una posible solución, aportando buenos resultados comparados con métodos convencionales en la sinterización de polvos metálicos [20]. Es de resaltar que el uso de DLA no ha sido ampliamente reportada en la literatura para la síntesis de materiales del tipo $\mathrm{Cu}-\mathrm{Ti}-\mathrm{TiC}$, y se desconocen las características finales de los productos con este tipo de procesamiento, pese a que su uso en materiales de matriz metálica se prevé como un importante aporte a la producción de partes en la metalurgia de polvos.

La presente investigación busca estudiar los efectos de la incorporación de diferentes fracciones de masa de titanio y un refuerzo constante de carburo de titanio en la porosidad y dureza en el sistema $\mathrm{Cu}$ - $\mathrm{Ti}$ TiC sinterizado con descarga luminiscente anormal.

\section{MATERIALES Y MÉTODOS}

Polvos de cobre (99\% de pureza), titanio $(99,5 \%$ de pureza) y carburo de titanio $(99,95 \%$ de pureza) fueron suministrados por Sigma-Aldrich, con tamaños de partícula promedio de $75 \mu \mathrm{m}, 60 \mu \mathrm{m}$ y $4 \mu \mathrm{m}$, respectivamente. Se elaboraron tres mezclas con diferentes contenidos en fracción de masa de titanio; $10 \%$, $15 \%$ y $20 \%$, manteniendo como refuerzo en cada una de ellas $1 \%$ de carburo de titanio. A fin de obtener una mezcla homogénea y evitar la aglomeración de los componentes, se realizaron suspensiones liquidas aforadas a $150 \mathrm{ml}$ de 2-propanol, las cuales se sometieron a baño ultrasónico $(40 \mathrm{~W})$ durante 10 minutos y agitación magnética a $1250 \mathrm{rpm}$ por 30 minutos con posterior secado a $50{ }^{\circ} \mathrm{C}$ por 1 hora. La homogeneidad de las mezclas fue verificada por medio de observación micrográfica usando un microscopio óptico Olympus GX41 con magnificación de 500X.

Los polvos mezclados se sometieron a compactación uniaxial en frio a $400 \mathrm{MPa}$ en una matriz de acero, obteniéndose probetas cilíndricas de $10 \mathrm{~mm}$ de diámetro y espesor promedio de $5 \mathrm{~mm}$. Las preformas en verde fueron sinterizadas por (DLA) cuyos parámetros de operación fueron: corriente directa-DC, presión de trabajo 2 Torr, atmósfera protectora de $\mathrm{Ar}$ y $\mathrm{N}_{2}$ con flujo constante de $90 \mathrm{ml} . \mathrm{min}^{-1}$ y $10 \mathrm{ml}^{. \mathrm{min}^{-1}}$ respectivamente. Voltaje de operación de $528 \mathrm{~V}$ con corriente de $310 \mathrm{~mA}$, tasa de calentamiento de $100{ }^{\circ} \mathrm{C} \cdot \mathrm{min}^{-1}$ y temperatura de sinterización de $850{ }^{\circ} \mathrm{C}$ por 30 minutos. Las muestras sinterizadas fueron enfriadas hasta $30{ }^{\circ} \mathrm{C}$ en la misma atmósfera de la cámara de sinterizado a fin de evitar la oxidación. La descripción del equipo de sinterizado se presenta en la Figura 1.

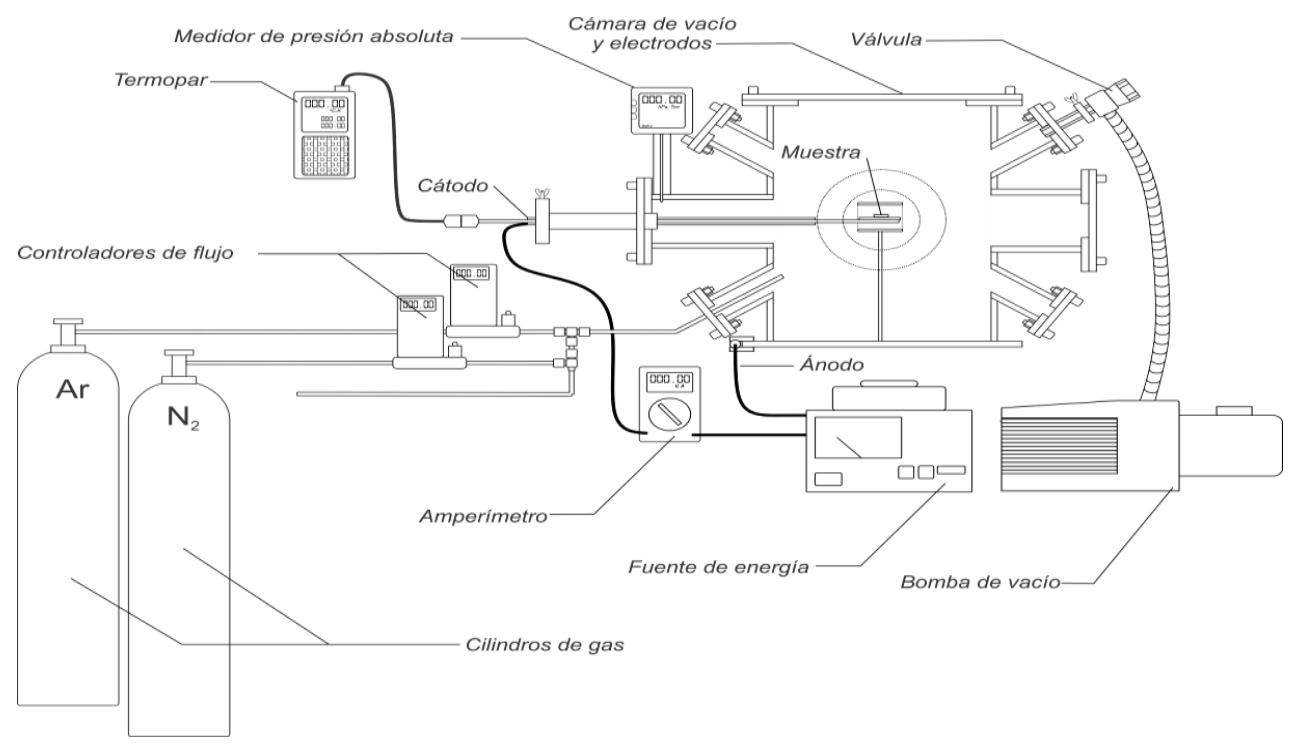

Figura 1: Esquema del equipo de sinterización por descarga luminiscente anormal (plasma). GSEC-1. 
Las preformas en verde y los sinterizados fueron observadas, a través, de microscopia electrónica de barrido en un equipo LEO 430 operado a $30 \mathrm{kV}$ usando los detectores de electrones secundarios-SE y electrones retrodispersados-BS. La caracterización composicional se realizó por medio de mapeo químico usando un detector EDS de Oxford instruments modelo 7059.

La porosidad de las muestras en verde y sinterizadas fue determinada por medio de análisis de las imágenes adquiridas por SEM usando el software Fiji (Image J versión 1.51), de acuerdo a los estándares de ASTM E1382 [21]. Alternativamente, se realizaron medidas de densidad por medio del método de Arquímedes bajo la norma ASTM B311 [22].

La difracción de rayos X (DRX) fue utilizada para determinar el tipo de fases presentes en los MMC; para esto se usó un equipo marca Pertpro PANalytical con longitud de onda de la línea característica del cobalto $\mathrm{K} \alpha=1,78901 \AA$. Los difractogramas fueron tomados utilizando la geometría Bragg-Brentano barriéndose el ángulo $2 \theta$ entre $20^{\circ}$ y $90^{\circ}$, con un paso de $0.013^{\circ}$, tiempo entre paso de 13,77 segundos, voltaje de operación de $40 \mathrm{kV}$ y corriente de $40 \mathrm{~mA}$. Posteriormente los MMC fueron sometidos a ensayo de microdureza Vickers en un microdurómetro Qualitest QV-1000AAT con carga de 100 gf y tiempo de permanencia de 15 segundos. Se tomaron 10 lecturas de cada muestra con fines estadísticos.

\section{RESULTADOS Y DISCUSIÓN}

\subsection{Caracterización morfológica}

Inicialmente los polvos: cobre, titanio y carburo de titanio fueron caracterizados por SEM para determinar su morfología y tamaño de partícula como se muestra en la Figura 2.
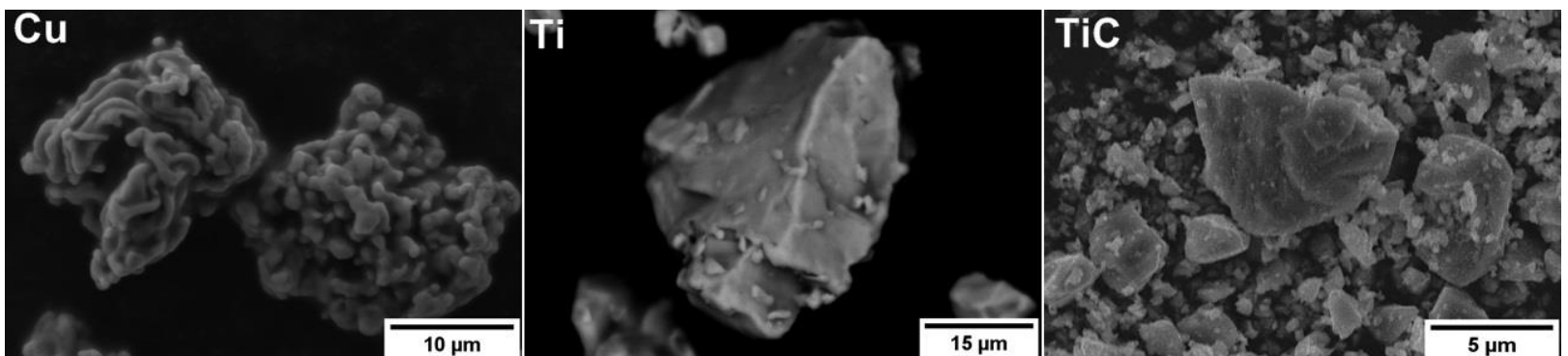

Figura 2: Imágenes por SEM usando detector de electrones secundarios. Morfología de los polvos empleados, a) Polvos de cobre, b) Polvos de titanio, c) Polvos de carburo de titanio.

Los polvos de cobre presentaron morfología alargada dendrítica en el rango de $14,1 \mu \mathrm{m}-29,8 \mu \mathrm{m}$, mientras que los polvos de titanio exhibieron una morfología angular con heterogeneidad en su tamaño de partícula en el intervalo de $9,73 \mu \mathrm{m}-45 \mu \mathrm{m}$ y el carburo de titanio presentó una formación angulosa con dimensiones de $1,33 \mu \mathrm{m}-3,99 \mu \mathrm{m}$.

En la Figura 3 se identifican los mapeos composicionales por SEM-EDS de los MMC sinterizados. La imagen de referencia (escala de grises) se obtuvo mediante SEM usando el detector de electrones retrodispersados con el fin de identificar las diferentes fases usando contraste Z. En la Figura 4, se muestran los difractogramas de rayos $\mathrm{X}$ de los compuestos sinterizados y los resultados son resumidos en la Tabla 1 .

La influencia del titanio en la formación de fases intermetálicas de cobre-titanio en el MMC por procesos difusivos se evidencia en las Figuras 3 y 4 . En la Figura 3 se observa que a medida que la fracción de masa de titanio empleada aumenta, se origina un trasporte activo de titanio y cobre de forma intergranular en los sinterizados. Los difractogramas exhiben que a medida que los niveles de titanio incrementan, se ve favorecida la formación de nuevas fases al interior del material. La Figura 4 a su vez evidencia que a niveles del $10 \%$ hay formaciones de intermetálicos mayoritarios como el $\mathrm{CuTi}_{2}$ en compañía de cobre, además, se presentan formaciones intermetálicas minoritarias como: $\mathrm{CuTi}_{3}$ y $\mathrm{Cu}_{3} \mathrm{Ti}$. En concentraciones superiores al $15 \%$ se describe la formación mayoritaria de $\mathrm{CuTi}_{2}$ y $\mathrm{Cu}_{3} \mathrm{Ti}$ acompañadas por las fases minoritarias: $\mathrm{Cu}_{4} \mathrm{Ti}_{3}$, $\mathrm{Ti}, \mathrm{Cu}$. En el caso del $15 \%$ de $\mathrm{Ti}$ se observa la presencia de la fase $\mathrm{CuTi}_{3}$, estos resultados pueden ser contrastados con las estructuras difusivas exhibidas por el titanio en el mapeo EDS (Figura 3). 


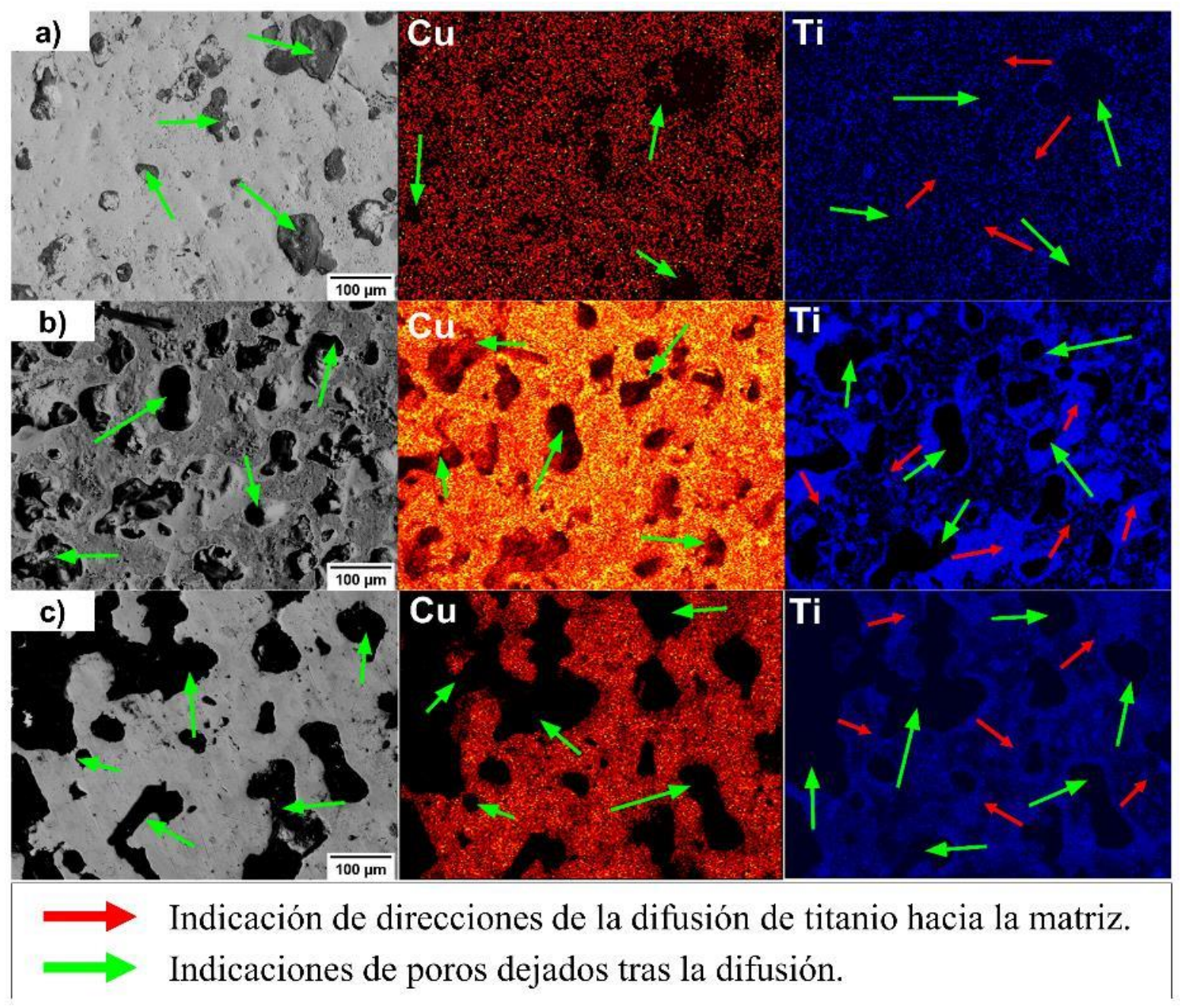

Figura 3: Mapeo composicional por EDS de los sinterizados con concentraciones del a) $10 \%$, b) $15 \%$ y c) $20 \%$ de titanio. Las flechas en verde evidencian las porosidades representativas en las muestras, y las flechas rojas el sentido propuesto de la difusión.

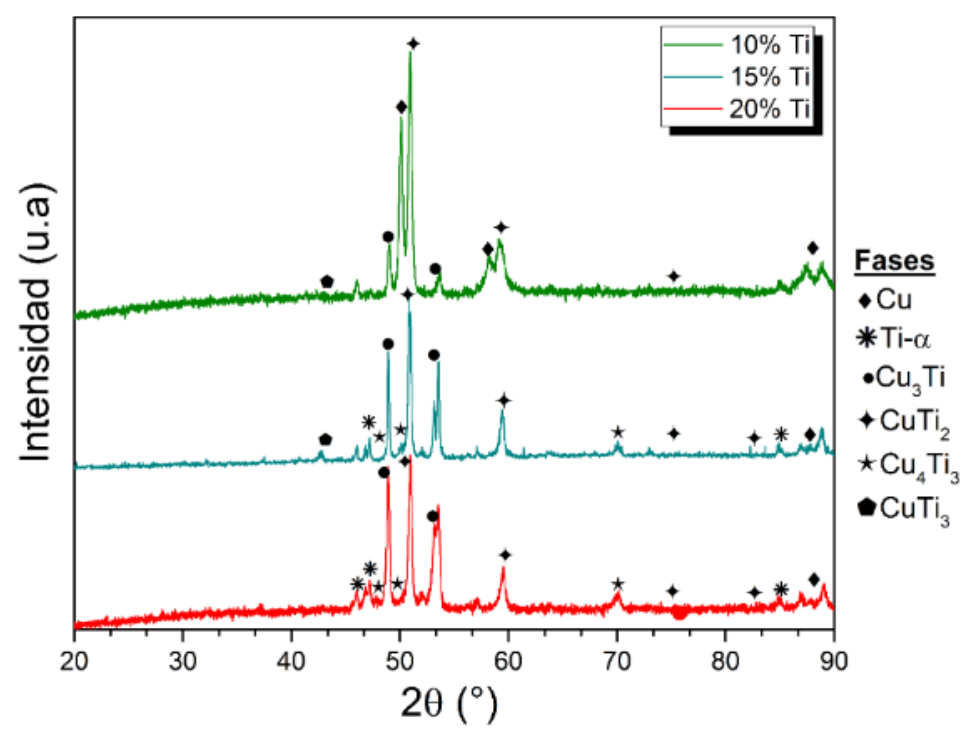

Figura 4: Difractogramas de los sinterizados. 
Tabla 1: Fases presentes en los diferentes sinterizados.

\begin{tabular}{l|l|l}
\hline MEZCLA & FASES MAYORITARIAS & FASES MINORITARIAS \\
\hline $10 \% \mathrm{Ti}$ & $\mathrm{CuTi}_{2}, \mathrm{Cu}$ & $\mathrm{Cu}_{3} \mathrm{Ti}_{1}, \mathrm{CuTi}_{3}$ \\
$15 \% \mathrm{Ti}$ & $\mathrm{Cu}_{3} \mathrm{Ti}_{1}, \mathrm{CuTi}_{2}$ & $\mathrm{Cu}_{4} \mathrm{Ti}_{3}, \mathrm{CuTi}_{3}, \alpha-\mathrm{Ti}, \mathrm{Cu}$ \\
$20 \% \mathrm{Ti}$ & $\mathrm{Cu}_{3} \mathrm{Ti}_{1}, \mathrm{CuTi}_{2}$ & $\mathrm{Cu}_{4} \mathrm{Ti}_{3}, \alpha-\mathrm{Ti}, \mathrm{Cu}$ \\
\hline
\end{tabular}

Las formaciones de las fases $\mathrm{Cu}_{4} \mathrm{Ti}_{3}$ y $\mathrm{Cu}_{3} \mathrm{Ti}$ son de carácter metaestable como lo describen las proyecciones termodinámicas de Zhu et al [23], pero la influencia del proceso energético que aporta el plasma es un mecanismo que favorece la formación de este tipo de fases. Se destaca que la fase $\mathrm{CuTi}_{3}$ es inestable, no obstante, se ha observado que su presencia es posible gracias al uso de sistemas de no equilibrio como el empleado en la presente investigación (DLA), su estabilidad está sujeta a la interacción que sufre con otras especies, su ausencia en concentraciones superiores al $20 \%$ es debida a la reacción de descomposición $\mathrm{CuTi}_{3}=\mathrm{CuTi}_{2}+\mathrm{Ti}$ [24], donde la presencia de titanio sin disolver, genera el desplazamiento de la reacción hacia la derecha $\left(\Delta \mathrm{G}_{\mathrm{r}}=-15,42 \mathrm{~kJ} \cdot \mathrm{mol}^{-1}\right)$ [25], [26].

La concentración energética, puntual y rápida debido a las altas tasas de calentamiento-enfriamiento del proceso de sinterización, así como la aplicación de altas presiones de compactación en frio, influenciaron en la formación de las respectivas fases metaestables en los sinterizados, favoreciendo la difusión atómica del sistema $\mathrm{Cu}$-Ti como se ha visto en la literatura [27]. Este tipo de procesos de formación por influencia de plasma ha sido estudiado por Chun Guo et al [28], donde se ha reportado que procesos con plasma puede ser un cooperador en la formación de fases en el sistema $\mathrm{Cu}$-Ti. Es de notar que el uso de DLA ha permitido obtener este tipo de fases con requerimientos de energía mucho menores $(0,163 \mathrm{~kW})$, a diferencia de investigaciones similares en el campo de los materiales compuestos $(3 \mathrm{~kW})$ [28]. Es razonable pensar que este tipo de formaciones favorece propiedades como la dureza [29], [30] y la conductividad eléctrica del material.

\subsection{Porosidad y densidad}

En la Figura 5 se presentan los resultados de densidad relativa debidas a la variación de la concentración de titanio, se caracteriza por una reducción de la densidad y un incremento de la aparición de porosidades en el material. Los valores de densidad relativa hallados en los diferentes MMC, indica que los aumentos en las fracciones de titanio generan una disminución de los valores de densidad de las preformas en verde, en razón a que el titanio es un metal ligero, que ante las prestaciones del sinterizado influye en la disminución de la densidad [31], siendo así que en fracciones de masa del $20 \%$ se obtienen valores bajos. Otro factor contribuyente de la disminución de la densidad está relacionado a los valores densidad que exponen los intermetálicos detectados en la difracción de rayos X.

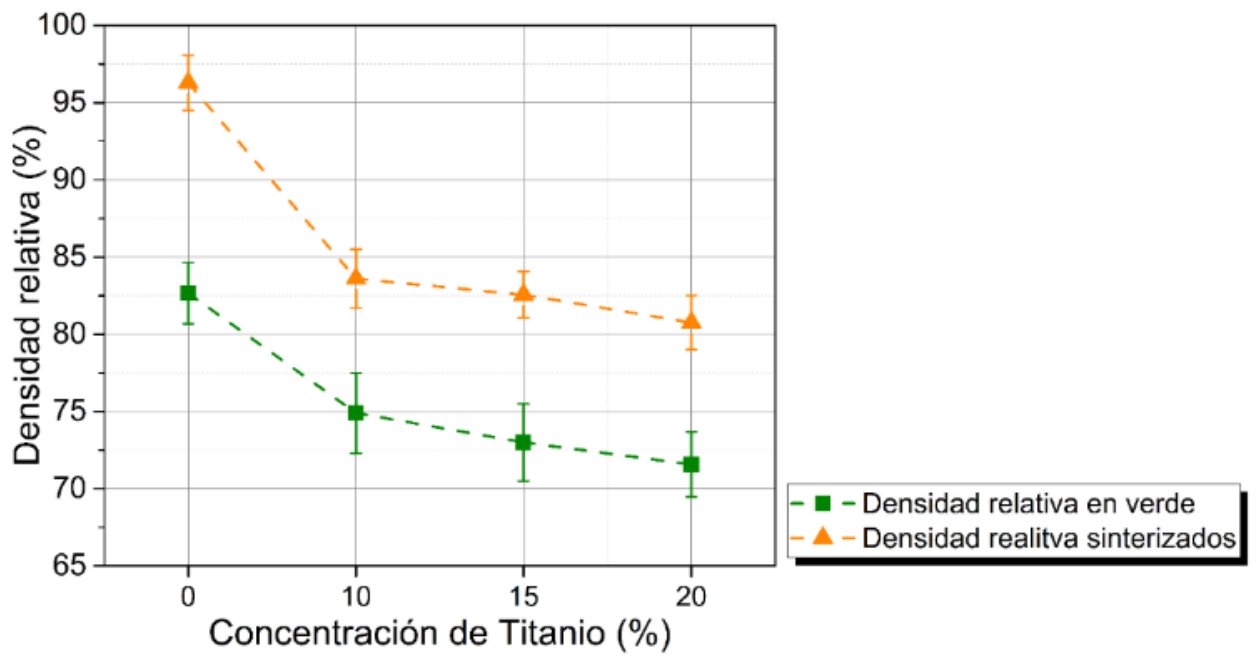

Figura 5: Densidad relativa en los MMC determinada por método de Arquímedes. 
Para las fases mayoritarias determinadas en este estudio (Figura 4), los valores de densidad teóricos reportadas en la literatura [32]-[34] son: $\mathrm{Cu}_{3} \mathrm{Ti}\left(7,79 \mathrm{~g} \cdot \mathrm{cm}^{-3}\right), \mathrm{CuTi}_{2}\left(5,66 \mathrm{~g} \cdot \mathrm{cm}^{-3}\right), \mathrm{Cu}_{4} \mathrm{Ti}_{3}\left(6,81 \mathrm{~g} \cdot \mathrm{cm}^{-3}\right), \mathrm{CuTi}_{3}$ $\left(5,54 \mathrm{~g} \cdot \mathrm{cm}^{-3}\right)$. Estos valores permiten inferir que la disminución de la densificación exhibida en la Figura 5 está relacionada con la precipitación de estas fases. La mayor fracción en masa de cobre en el compuesto con $10 \%$ Ti presentó un mayor grado de densificación, a pesar de la presencia de la fase $\mathrm{CuTi}_{2}$ menos densa, que debido a su formación tiende a generar la porosidad expuesta en esta concentración. En los contenidos superiores al $15 \%$ de titanio se ha observado que la combinación de $\mathrm{Cu}_{3} \mathrm{Ti}$ y $\mathrm{CuTi}_{2}$ tienden a disminuir de manera acentuada la densidad del material, en razón a que estas fases son de estructura ortorrómbica y tetragonal. Las cuales en virtud de su cristalografía generan la formación de porosidad, ya que estas fases durante el proceso de sinterizado expanden el material al ocupar un mayor volumen; observándose en el $\mathrm{Cu}_{3} \mathrm{Ti}$ valores cercanos a $104,94 \AA^{3}[35]$ y $92,48 \AA^{3}$ [35] en el caso del $\mathrm{CuTi}_{2}$, en comparación a las materias principales de la matriz cobre-titanio que exhiben volúmenes de 47,67 $\AA^{3}$ [33] y 35,46 $\AA^{3}$ [33], respectivamente. Esto demuestra que la precipitación intermetálica tiene un papel importante en la formación de poro, debido a que el volumen que ocupan es mucho mayor en comparación a las fases en verde.

De acuerdo a esto, la contracción durante el enfriamiento no es la suficiente para compensar el cambio de volumen y el transporte de masa de las reacciones de formación de los intermetálicos, de modo que dejan porosidad que incrementa el volumen total final del compuesto. Al seguir este proceso es presumible que la inclusión de contenidos de titanio mayores permitirá obtener una inclinación de las reacciones de difusión hacia fases cada vez menos densas con estructuras del tipo ortorrómbico, que en el espectro de intermetálicos del sistema exhiben los menores valores de densidad y energía de Gibbs [33].

Tabla 2: Porosidad superficial registrada en las muestras por medio de análisis de imágenes.

\begin{tabular}{l|l|l}
\hline \multirow{2}{*}{ MUESTRA } & $\begin{array}{l}\text { POROSIDAD EN } \\
\text { VERDE (\%) }\end{array}$ & $\begin{array}{l}\text { POROSIDAD EN } \\
\text { SINTERIZADOS (\%) }\end{array}$ \\
\hline Blanco & 9,15 & 0,76 \\
$10 \%$ de Ti & 7,21 & 9,61 \\
$15 \%$ de Ti & 6,18 & 11,25 \\
$20 \%$ de Ti & 5,20 & 13,61 \\
\hline
\end{tabular}

Tabla 3: Tamaño de poro promedio y factor de forma (circularidad).

\begin{tabular}{l|l|l}
\hline MUESTRA & TAMAÑO PROMEDIO (mm) & CIRCULARIDAD (u.a) \\
\hline $10 \%$ de Ti & 30,20 & 0,864 \\
$15 \%$ de $\mathrm{Ti}$ & 43,40 & 0,800 \\
$20 \%$ de Ti & 127,4 & 0,784 \\
\hline
\end{tabular}

La Figura 6 evidencia un ejemplo del tipo de resultados obtenidos en el análisis de imágenes para la determinación de los valores de porosidad recopilados en la Tabla 2. Se observa que a medida que la fracción de $\mathrm{Ti}$ aumenta en las preformas en verde la porosidad disminuye, mientras que en los compuestos sinterizados aumenta. Este efecto se presenta debido a que en el proceso compresivo en la manufactura de las preformas, el titanio actúa como generador de centros duros donde el cobre tiende a deformarse sobre esta superficie. Esta deformación localizada es aprovechada por la morfología del cobre (Figura 2) que permite una mayor área de cubrimiento entre las partículas, lo que explica la disminución de la porosidad en verde, generado por la movilidad y distribución de los polvos de cobre. De acuerdo con Atiyah et al [36] la presión de compactación tiene un efecto esencial en la disminución del porcentaje de porosidad y configuración de fases intermetálicos en sistemas pulvimetalúrgicos similares, tales como los compuestos por la combinación 
de materias primas de naturaleza cúbica centrada en las caras (elementos como níquel y cobre en mayor proporción) y agregados hexagonales (titanio).

La porosidad en los MMC es influenciada por el proceso de sinterizado de la siguiente manera: la configuración de poro de mayor tamaño en las mezclas de $10 \%, 15 \%$ y $20 \%$ de Ti, se debe a la contribución de las fases $\mathrm{CuTi}_{2}$ y $\mathrm{Cu}_{3} \mathrm{Ti}$, respectivamente, lo cual está relacionado a una alta reacción del titanio (granos primarios) con el cobre. Esta reacción deja poros en el proceso de síntesis debido a fenómenos asociados a la formación de intermetálicos, los cuales generan al final del proceso macro poros que no son llenados con material, promovido por la alta velocidad del proceso y la menor densidad de las nuevas fases formadas. Estas fases no tienen la suficiente energía para trasportar su masa hasta los espacios dejados por las partículas de titanio debido a que parte de la energía se ha consumido en la formación de las mismas y en el crecimiento de grano. Los granos intermetálicos no crecen lo suficiente para llenar las cavidades de poro en razón del acelerado trasporte de titanio hacia el interior del compuesto.

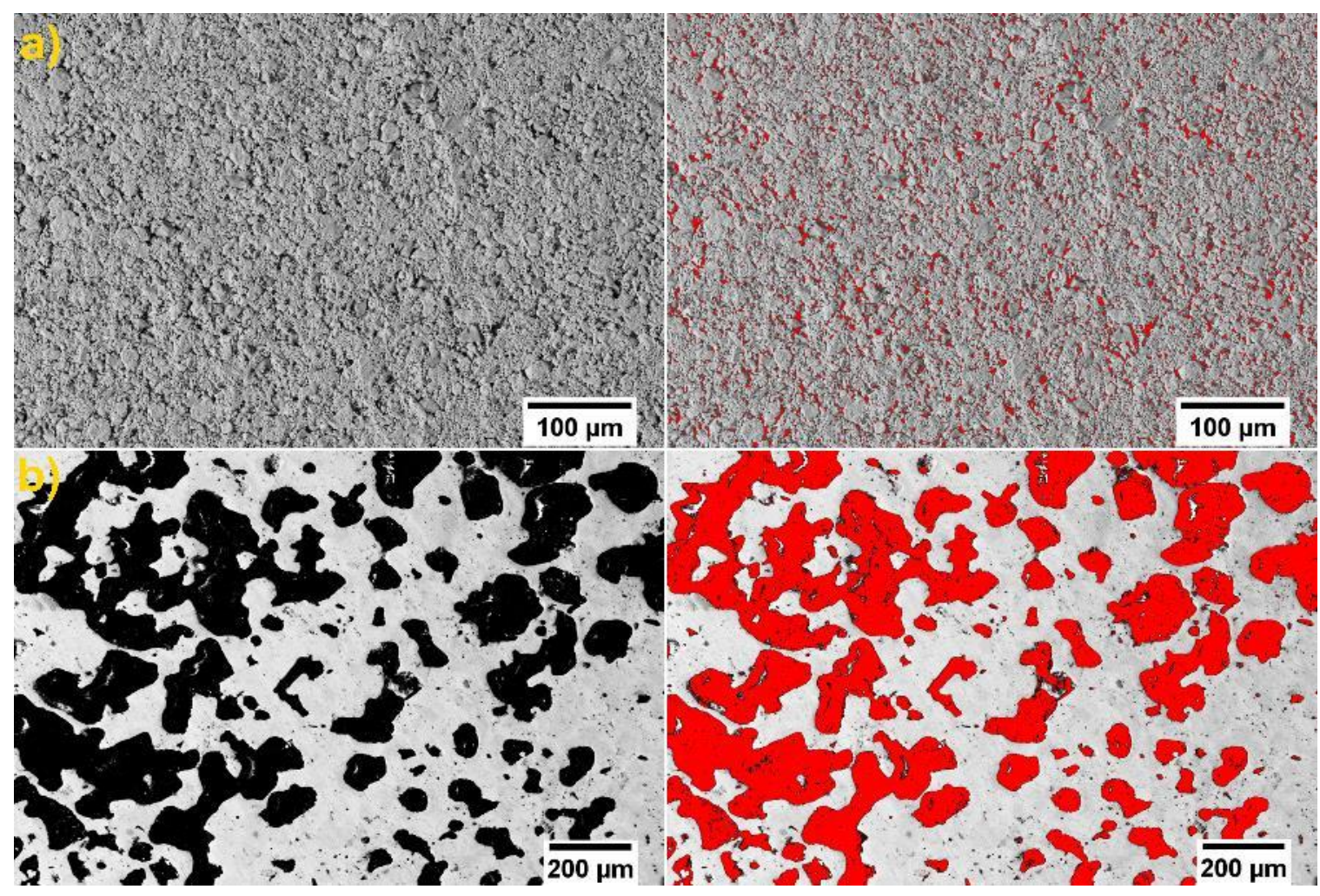

Figura 6: Muestra representativa de la determinación de la porosidad por medio del análisis de imágenes. Tomando como referencia la mezcla al $20 \%$ de $\mathrm{Ti}$ a) en verde y b) sinterizada. A la derecha se presenta la imagen binaria para la cuantificación y distribución de poros.

La mezcla con $10 \%$ de Ti evidenció la formación de fases intermetálicas debido a la rápida combinación del titanio con el cobre, originando poros en el material debido a la formación de fases intermetálicas en esas regiones (ver flechas verdes en la Figura 3). Este fenómeno es aumentado con la aparición de las fases $\mathrm{Cu}_{3} \mathrm{Ti}, \mathrm{CuTi}_{2}$ a partir del $15 \%$ de Ti en el compuesto. En concentraciones del $20 \%$ de Ti la porosidad es mayor que en las otras mezclas y el tamaño de poro sufre un acelerado crecimiento con respecto a los menores porcentajes de titanio. Este fenómeno es debido a que en concentraciones de titanio menores al 20\%, se originan zonas con material insuficiente para seguir formando fases intermetálicas como el $\mathrm{CuTi}_{2}, \mathrm{Cu}_{3} \mathrm{Ti}$ y fases masivas como el $\mathrm{Cu}_{4} \mathrm{Ti}_{3}$, configurando una deformación de los poros como se describe en los valores de factor de forma (Tabla 3 ).

La naturaleza desigual de los componentes $\mathrm{Cu}$-Ti no presentan las condiciones idóneas para su reacción con el cobre a causa de la presencia de la capa pasiva en el titanio y la desigual estructura cristalina [37], sin embargo, se evidencia al observar la Figura 3 la difusión desde el titanio hacia la matriz de cobre, la cual generó la formación de porosidad en el material, en razón a la rápida reacción del titanio con el cobre, y el lento desplazamiento de los productos durante la consolidación del material. Basados en los cálculos 
expuestos por Laik et al [24] se ha demostrado que a temperaturas de $850{ }^{\circ} \mathrm{C}$ es posible obtener una difusión de titanio (BCC) hacia el cobre (FCC) con una difusividad de $4,85 \times 10^{-14} \mathrm{~m}^{2} . \mathrm{s}^{-1}$. Esto es debido a la trasformación del titanio alfa en titanio beta en este rango de temperatura, lo cual promueve la movilidad atómica y los mecanismos de reacción intermetálica, en contraposición a los cálculos de difusión de cobre hacia titanio (BCC) propuestos por Lee et al [38] de cerca de $3,53 \times 10^{-16} \mathrm{~m}^{2} . \mathrm{s}^{-1}$. Un factor adicional que puede ser tenido en cuenta es la orientación de los límites de grano y la morfología de las materias de partida. Se ha observado que la morfología angulosa favorece las reacciones de precipitación en las interfases $\mathrm{Cu}-\mathrm{Ti}$ en razón a la preferencia de la nucleación de intermetálicos en zonas de alta aleatoriedad, lo cual esta correlacionada con el carácter y la estructura del límite del grano que se imprime durante la deformación en la compactación y a la posterior inclusión de cargas eléctricas no orientas durante la descarga del plasma, promoviendo la energía y modificación difusiva como ha sido descrito para este sistema [39].

\subsection{Microdureza}

En la Figura 7 se aprecia la dureza alcanzada en los MMC, a medida que la cantidad de masa de titanio aumenta se ha generado una mayor dureza del material, esto es debido a varios factores en los que el incremento de fases intermetálicas implica un mejoramiento paulatino de esta propiedad. Los mecanismos de reacción en estado sólido del $\mathrm{Cu}$ y el Ti debido a la diferencia en el radio atómico de las especies [40] 1,45 para el Cu y 1,76 A para el Ti crean esfuerzos de tensión en la matriz de cobre, que devienen en la restricción de los planos de deslizamientos naturales de la fase FCC de la matriz.

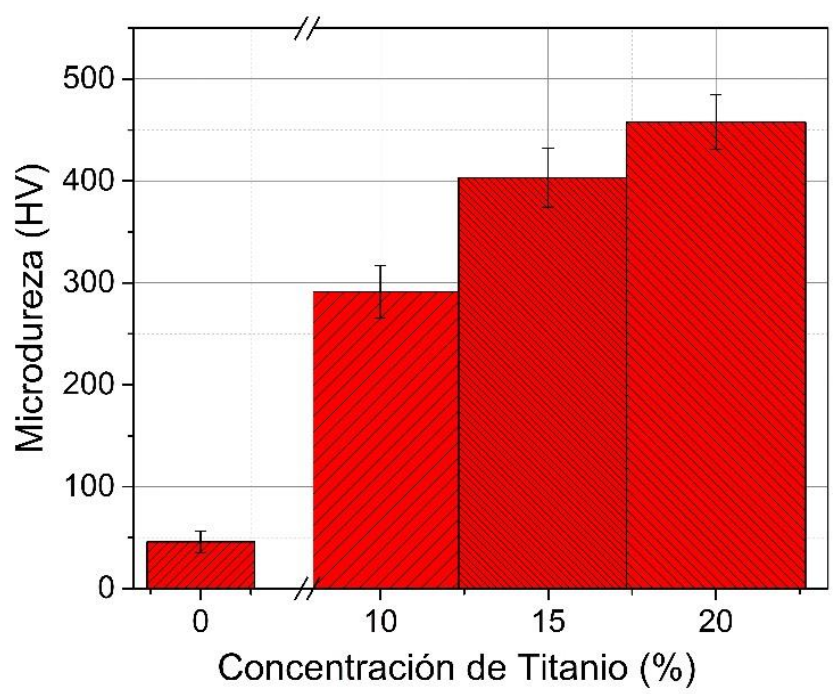

Figura 7: Microdurezas determinadas bajo las diferentes concentraciones de titanio.

La aparición de los intermetálicos $\mathrm{Cu}_{3} \mathrm{Ti}$ y el $\mathrm{CuTi}_{2}$ de manera paulatina explican los valores determinados ya que sus durezas son cercanas a estudios similares dentro del sistema, ya que estas fases poseen un valor intermedio entre las características metálicas y cerámicas, en las que el alto grado covalente de sus enlaces generan una limitación de la energía de deformación a causa del limitado número de planos de deslizamiento y al grado de anisotropía que presentan estas fases [33]. Además, este comportamiento se obedece al alto módulo de elasticidad de estas fases siendo reportados [32] valores de hasta 138,94 GPa para el $\mathrm{Cu}_{3} \mathrm{Ti}$ y $141,95 \mathrm{GPa}$ para el $\mathrm{CuTi}_{2}$ en comparación a las fases $\mathrm{Cu}(128,35 \mathrm{GPa})$ [41] y Ti (116 GPa) [42].

Otro factor a considerar en el aumento de la dureza del material es la presencia del refuerzo TiC, por ser una fase cerámica de elevada dureza, mostrando una cierta influencia en el sistema de estudio $\mathrm{Cu}$-Ti-TiC, que incrementa el comportamiento mecánico de este tipo de MMC [43]. Teniendo en cuenta las altas fracciones de porosidad, puede considerarse que los valores de dureza pueden alcanzar valores mayores por medio de tratamientos térmicos o termomecánicos posteriores con el fin de sellar los poros en los sinterizados.

\section{CONCLUSIONES}

Los resultados experimentales evidencian que el incremento de titanio influye en la porosidad de los MMC sin afectar las propiedades mecánicas como dureza, aportando conocimientos acerca del sistema $\mathrm{Cu}-\mathrm{Ti}$ - $\mathrm{TiC}$ bajo las condiciones de síntesis aplicadas, brindando la posibilidad de incorporar altas fracciones 
volumétricas de refuerzo metálico con el propósito de propiciar la mejora de propiedades. En los compuestos con contenidos de titanio del $10 \%$ y $15 \%$ se detectó la fase $\mathrm{CuTi}_{3}$. Además, en todas las concentraciones se observó la presencia mayoritaria de las formaciones $\mathrm{Cu}_{3} \mathrm{Ti}$ y $\mathrm{CuTi}_{2}$ generadas por la conjunción de los efectos de los procesos de compactación y de sinterización, los cuales favorecieron la alta difusión de Ti propiciando la aparición de esta clase de intermetálicos. Las fases intermetálicas $\mathrm{Cu}_{3} \mathrm{Ti}_{\text {y }} \mathrm{CuTi}_{2}$ sugiere que tienen influencia en el aumento de la dureza del MMC obtenida con contenidos superiores al 15\% de Ti. Finalmente, los resultados exponen que un control de la microestructura y del proceso debe tenerse en cuenta en la síntesis de esta clase de materiales a fin de incrementar su uso en el campo ingenieril especialmente en la industria automovilística y biomédica donde promete grandes beneficios en razón al equilibrio entre el costo y beneficio que se logra alcanzar con el uso de este sistema.

\section{BIBLIOGRAFÍA}

[1] ICSG, "International copper study group". [En línea]. Disponible en: www.icsg.org. [Consultado: 19-oct2018].

[2] ALANEME, K. K., ODONI, B. U., "Mechanical properties, wear and corrosion behavior of copper matrix composites reinforced with steel machining chips", Engineering Science and Technology, an International Journal, v. 19, n. 3, pp. 1593-1599, sep. 2016.

[3] HAMADA, A. S., KHOSRAVIFARD, A., KISKO, A. P., et al., "High temperature deformation behavior of a stainless steel fiber-reinforced copper matrix composite", Materials Science and Engineering: A, v. 669, pp. 469-479, jul. 2016.

[4] JENA, P., BROCCHI, E., MOTTA, M., "In-situ formation of $\mathrm{Cu}-\mathrm{Al} 2 \mathrm{O} 3$ nano-scale composites by chemical routes and studies on their microstructures", Materials Science and Engineering: A, v. 313, n. 1-2, pp. 180-186, ago. 2001.

[5] THANKACHAN, T., PRAKASH, K. S., "Microstructural, mechanical and tribological behavior of aluminum nitride reinforced copper surface composites fabricated through friction stir processing route", Materials Science and Engineering: A, v. 688, pp. 301-308, mar. 2017.

[6] NAYAK, D., DEBATA, M., "Effect of composition and milling time on mechanical and wear performance of copper-graphite composites processed by powder metallurgy route", Powder Metallurgy, v. 57, núm. 4, pp. 265-273, sep. 2014.

[7] SATHISKUMAR, R., MURUGAN, N., DINAHARAN, I., et al., "Characterization of boron carbide particulate reinforced in situ copper surface composites synthesized using friction stir processing", Materials Characterization, v. 84, pp. 16-27, oct. 2013.

[8] LI, M., et al., "Tensile behavior and strengthening mechanism in ultrafine TiC0.5 particle reinforced $\mathrm{Cu}-$ Al matrix composites", Journal of Alloys and Compounds, v. 628, pp. 186-194, abr. 2015.

[9] NEMATI, N., KHOSROSHAHI, R., EMAMY, M., et al., "Investigation of microstructure, hardness and wear properties of $\mathrm{Al}-4.5 \mathrm{wt} . \% \mathrm{Cu}-\mathrm{TiC}$ nanocomposites produced by mechanical milling", Materials \& Design, v. 32, n. 7, pp. 3718-3729, ago. 2011.

[10] BUYTOZ, S., DAGDELEN, F., ISLAK, S., et al., "Effect of the TiC content on microstructure and thermal properties of $\mathrm{Cu}-\mathrm{TiC}$ composites prepared by powder metallurgy", Journal of Thermal Analysis and Calorimetry, v. 117, n. 3, pp. 1277-1283, sep. 2014.

[11] BAGHERI, G. A., "The effect of reinforcement percentages on properties of copper matrix composites reinforced with TiC particles", Journal of Alloys and Compounds, vol. 676, pp. 120-126, ago. 2016.

[12] AKBARPOUR, M. R., SALAHI, E., HESARI, F. A., et al., "Microstructural development and mechanical properties of nanostructured copper reinforced with $\mathrm{SiC}$ nanoparticles", Materials Science and Engineering: A, v. 568, pp. 33-39, abr. 2013.

[13] AKBARPOUR, M. R., HESARI, F. A., "Characterization and hardness of TiCu-Ti $2 \mathrm{Cu} 3$ intermetallic material fabricated by mechanical alloying and subsequent annealing", Materials Research Express, v. 3, núm. 4, p. 045004, abr. 2016.

[14] PINA, V. G., AMIGÓ, V., MUÑOZ, A.I, "Microstructural, electrochemical and tribo-electrochemical characterisation of titanium-copper biomedical alloys", Corrosion Science, v. 109, pp. 115-125, ago. 2016. 
[15] LI, J., et al., "Optimized thermal properties in diamond particles reinforced copper-titanium matrix composites produced by gas pressure infiltration”, Composites Part A: Applied Science and Manufacturing, v. 91, pp. 189-194, dic. 2016.

[16] WANG, F., LI, Y., WANG, X., et al., "In-situ fabrication and characterization of ultrafine structured $\mathrm{Cu}-\mathrm{TiC}$ composites with high strength and high conductivity by mechanical milling", Journal of Alloys and Compounds, vol. 657, pp. 122-132, feb. 2016.

[17] WANG, F., et al., "Investigation on hot deformation behavior of nanoscale TiC-strengthened Cu alloys fabricated by mechanical milling”, Materials Science and Engineering: A, v. 668, pp. 1-12, jun. 2016.

[18] MORARU, C. G., POP, M. A., BEDO, T., et al., "Spark plasma sintering of novel copper based metal matrix composites", Metalurgia, v. 65, n. 6, pp. 38-43, 2013.

[19] SAMAL, C. P., PARIHAR, J. S., CHAIRA, D., "The effect of milling and sintering techniques on mechanical properties of $\mathrm{Cu}$-graphite metal matrix composite prepared by powder metallurgy route", Journal of Alloys and Compounds, v. 569, pp. 95-101, ago. 2013.

[20] PÉREZ-VELÁSQUEZ, S., PINEDA-TRIANA, Y., AGUILAR-CASTRO, Y., et al., "Efecto del porcentaje de refuerzo frente al desgaste en compuestos de matriz metálica sinterizados con descarga luminiscente anormal”, Revista de Metalurgia, v. 52, n. 1, p. e059, mar. 2016.

[21] ASTM E1382-97, "Standard Test Methods for Determining Average Grain Size Using Semiautomatic and Automatic Image Analysis", 2015.

[22] ASTM B-311, "Standard Test Method for Density of Powder Metallurgy ( PM ) Materials Containing Less Than Two Percent Porosity 1”, ASTM International, v. 93, n. Reapproved, pp. 1-5, 2008.

[23] ZHU, Y. D., YAN, M. F., ZHANG, Y. X., et al., "First-principles investigation of structural, mechanical and electronic properties for $\mathrm{Cu}-\mathrm{Ti}$ intermetallics", Computational Materials Science, v. 123, pp. 70-78, oct. 2016.

[24] LAIK, A., BHANUMURTHY, K., KALE, G. B., et al., "Diffusion characteristics in the Cu-Ti system", International Journal of Materials Research, v. 103, n. 6, pp. 661-672, jun. 2012.

[25] YAN, M. F., ZHU, Y. D., ZHANG, C. S., et al., "Microstructure and mechanical properties of coppertitanium-nitrogen multiphase layers produced by a duplex treatment on C17200 copper-beryllium alloy", Materials \& Design, v. 84, pp. 10-17, nov. 2015.

[26] CANALE, P., SERVANT, C., "Thermodynamic Assessment of the Cu-Ti System Taking into Account the New Stable Phase CuTi 3", Zeitschrift für Metallkunde, v. 93, n. 4, pp. 273-276, abr. 2002.

[27] ZHANG, J. Y., et al., "Alloying effects on the microstructure and mechanical properties of nanocrystalline $\mathrm{Cu}$-based alloyed thin films: Miscible $\mathrm{Cu}-\mathrm{Ti}$ vs immiscible $\mathrm{Cu}-\mathrm{Mo}$ ”, Acta Materialia, v. 143, pp. 55-66, ene. 2018.

[28] GUO, C., ZHOU, J., YU, Y., et al., "Microstructure and tribological properties of Ti-Cu intermetallic compound coating”, Materials \& Design (1980-2015), v. 36, pp. 482-489, abr. 2012.

[29] TIRTH, V., "Dry Sliding Wear Behavior of 2218 Al-Alloy-Al 2 O 3 (TiO 2 ) Hybrid Composites", Journal of Tribology, v. 140, n. 2, p. 021603, sep. 2017.

[30] SINGH, S. K., SINGH, S., KUMAR, A., et al., "Thermo-mechanical behavior of TiO2 dispersed epoxy composites", Engineering Fracture Mechanics, v. 184, pp. 241-248, oct. 2017.

[31] SHAHEDI ASL, M., SABAHI NAMINI, A., MOTALLEBZADEH, A., et al., "Effects of sintering temperature on microstructure and mechanical properties of spark plasma sintered titanium", Materials Chemistry and Physics, v. 203, pp. 266-273, ene. 2018.

[32] ZHU, Y. D., YAN, M. F., ZHANG, Y. X., et al., "First-principles investigation of structural, mechanical and electronic properties for $\mathrm{Cu}-\mathrm{Ti}$ intermetallics", Computational Materials Science, v. 123, pp. 70-78, oct. 2016.

[33] CHEN, S., DUAN, Y. H., HUANG, B., et al., "Structural properties, phase stability, elastic properties and electronic structures of $\mathrm{Cu}-\mathrm{Ti}$ intermetallics", Philosophical Magazine, v. 95, n. 32, pp. 3535-3553, nov. 2015.

[34] YANG, J., HUANG, J., YE, Z., et al., "First-principles calculations on structural energetics of Cu-Ti binary system intermetallic compounds in $\mathrm{Ag}-\mathrm{Cu}-\mathrm{Ti}$ and $\mathrm{Cu}-\mathrm{Ni}-\mathrm{Ti}$ active filler metals", Ceramics International, v. 43, n. 10, pp. 7751-7761, jul. 2017. 
[35] GHOSH, G., "First-principles calculations of structural energetics of $\mathrm{Cu}-\mathrm{TM}$ (TM=Ti, Zr, Hf) intermetallics", Acta Materialia, v. 55, n. 10, pp. 3347-3374, jun. 2007.

[36] ATIYAH, A. A., ALI, A. R. K. A., DAWOOD, N. M., "Characterization of NiTi and NiTiCu Porous Shape Memory Alloys Prepared by Powder Metallurgy (Part I)", Arabian Journal for Science and Engineering, v. 40, n. 3, pp. 901-913, mar. 2015.

[37] SHON, J., PARK, J., CHO, K., et al., "Effects of various sintering methods on microstructure and mechanical properties of CP-Ti powder consolidations", Transactions of Nonferrous Metals Society of China, v. 24, n. SUPPL. 1, pp. s59-s67, jul. 2014.

[38] LEE, S.Y., IIJIMA, Y., TAGUCHI, O., et al., "Diffusion of Copper and Silver in Beta Titanium", Journal of the Japan Institute of Metals, v. 54, n. 5, pp. 502-508, 1990.

[39] SEMBOSHI, S., SATO, M., KANENO, Y., IWASE, A., TAKASUGI, T., "Grain Boundary Character Dependence on Nucleation of Discontinuous Precipitates in Cu-Ti Alloys", Materials, v. 10, n. 4, p. 415, abr. 2017.

[40] CLEMENTI, E., RAIMONDI, D. L., REINHARDT, W. P., “Atomic Screening Constants from SCF Functions. II. Atoms with 37 to 86 Electrons", The Journal of Chemical Physics, v. 47, n. 4, pp. 1300-1307, ago. 1967.

[41] RASSOULINEJAD-MOUSAVI, S. M., MAO, Y., ZHANG, Y., "Evaluation of copper, aluminum, and nickel interatomic potentials on predicting the elastic properties", Journal of Applied Physics, v. 119, n. 24, p. 244304 , jun. 2016.

[42] RISTIĆ, R., et al., "Properties and atomic structure of amorphous early transition metals", Journal of Alloys and Compounds, v. 504, n. SUPPL. 1, pp. S194-S197, ago. 2010.

[43] MARKANDEYA, R., NAGARJUNA, S., SARMA, D. S., "Precipitation hardening of Cu-Ti-Cr alloys", Materials Science and Engineering: A, v. 371, n. 1-2, pp. 291-305, abr. 2004.

\section{ORCID}

Cristian Daniel Bohórquez Moreno Sandra Patricia Pérez Velásquez Armando Sarmiento Santos Martin Emilio Mendoza Oliveros https://orcid.org/0000-0003-0328-3336

https://orcid.org/0000-0002-0211-1519

https://orcid.org/0000-0003-3382-0104

https://orcid.org/0000-0002-2999-5166 